\title{
Porous Polymeric Films from Microbubbles Generated Using a T-junction Microfluidic Device
}

\author{
M. Elsayed, A. Kothandaraman, M. Edirisinghe, J. Huang* \\ Department of Mechanical Engineering, University College London, Torrington Place, \\ London WC1E 7JE, UK
}

\section{Corresponding Author}

*E-mail: jie.huang@ucl.ac.uk. Tel: +4420 76797183.

\begin{abstract}
In this work, a simple microfluidic junction with a T-geometry and coarse (200 $\mu \mathrm{m}$ diameter) capillaries was used to generate monodisperse microbubbles with an alginate polymer shell. Subsequently, these bubbles were used to prepare porous alginate films with good control over the pore structure. The lack of pore size, shape, and surface control in scalable forming of polymeric films is a major application-limiting drawback at present. Controlling the thinning process of the shell of bubbles to tune the surface of the resulting structures was also explored. Films were prepared with nano-patterned surfaces by controlling the thinning of the bubble shell, with the aid of surfactants, to induce efficient bursting (fragmentation) of bubbles to generate nano-droplets which become embedded within the film surface. This novel feature greatly expands and enhances the use of hydrophilic polymers in a wide range of biomedical applications, particularly in drug delivery and tissue engineering, such as studying cellular responses to different morphological surfaces.
\end{abstract}

Keywords: microbubbles; microfluidic; polymer; films 


\section{Introduction}

There is a significant demand for highly ordered biocompatible and biodegradable porous structures for drug delivery and tissue engineering applications (1). Porous scaffolds, in particular, have received much attention as a result of their usefulness in various applications, including the immobilization of biomolecules and cells (2). Polymeric scaffolds and films have unique advantages for certain drug delivery systems, such as liquid and semisolid drug delivery products, because they provide a larger surface area of application, added to the ability to be designed to adhere to certain types of biological tissues (3). Additionally, controlling the porosity is of great importance in the area of tissue engineering, because larger pores are capable of allowing cells to be seeded within the scaffold, while smaller pores can inhibit this process and inhibit cell infiltration (4). Porous structures with a larger pore size distribution and different pore shapes make it difficult to conduct systematic investigations for cellular activities and interactions, including cell signaling (5). Scaffolds with more spherical pores have better mechanical properties and resist compressive stresses compared to scaffolds with irregular pores (6).

Various methods have been developed to prepare porous structures, including freeze-drying, electrospraying, particle leaching, and bioprinting (7). However, the resulting scaffolds contained a wide pore size distribution. The ability to incorporate biomolecules (such as enzymes) into the porous films produced by multi-step methods, such as breath figure (8), can be greatly limited, since organic solvents (such as dichloromethane and carbon disulfide) are involved in the preparation process (9), which often damage the biomolecules. On the other hand, the more simplified (one-step) methods, such as the conventional casting of films (3) produce porous films with non-controlled pore sizes (10). As a result, we believe that there is a pressing need for developing new methods for the preparation of porous structures with controlled porosity, without involving harsh conditions, such as the involvement of organic solvents, heat treatments, etc. This would expand their biomedical applications, especially when sensitive biomolecules are involved. Furthermore, this would increase the utilization of a wide category of polymers, i.e., natural polymers, which lose their internal structure as a result of harsh processing.

Natural polymers are characterized by a highly organized chemical structure, which, if preserved, can add additional desirable features to their therapeutic use (11). In this regard, microbubbles are regarded as a powerful tool for the preparation of polymeric devices for 
various biomedical uses, such as drug delivery systems (3) and tissue engineering scaffolds (12). Monodisperse microbubbles are obtained using a one-step microfluidic-based method, in contrast to other conventional preparation methods, such as sonication (13). The microbubble formation process using a T-junction occurs in three stages: the growth stage, the necking stage, and finally the pinch off stage (14). The gas and liquid phases meet at the junction, and a column of gas starts to form and expand until the bubble finally separates.

Despite the novelty of the microbubbling technique, polymer tissue engineering scaffolds prepared in this way have some limitations. Ekemen et al. (15) produced silk fibroin polymer films using elecrohydrodynamic bubbling that were characterized by undefined/folded pore shape and large size distribution. In this regard, Zmora et al. (6) found that scaffolds with spherical pores are capable of resisting compressive stresses, compared to those with elongated pores. They also discovered that the architecture of the pore significantly affects the morphology of the seeded cells (hepatocytes). To the best of our knowledge, few studies engaged the utilization of the process of bubble bursting in controlling the shape of the pores, and this is mainly due to the challenges involved in understanding the process of bubble bursting (16). Our previous studies investigated the different factors that influence the process of bubble bursting, such as the bubble size and bubbling solution properties, and lead to the utilization of the process to obtain hydrophilic alginate nanoparticles (17). Thus, the present research aims to use microbubbles, prepared using a gas pressure-driven T-junction, to produce uniform porous structures in a controlled manner, with uniform pore size and shape, through using the process of bubble bursting. The produced structures can be used for drug delivery and other tissue engineering applications. Additionally, such a technique can offer a way to produce multifunctional porous structures with surface-embedded nanoparticles by controlling the formation of nano-droplets and inducing the efficient bursting of bubbles using a one-step processing method. We believe that this feature is highly useful in applications that require particular surface properties, which have been found to noticeably change the surrounding cellular behavior (18).

Polymeric solutions are used to prepare the microbubbles, a combination of a biocompatible polymer (alginate), phospholipids (L- $\alpha$-phosphatidylcholine), and a surfactant polyethylene glycol-40- stearate (PEG-40S). Alginate is a biocompatible polymer known to enhance the bioavailability of drugs (19), and, when cross-linked with certain cations (such as aluminum, copper, and zinc), can possess antimicrobial properties (20). Alginate systems are capable of 
immobilizing a large volume of therapeutic agents easily diffused to the surrounding medium (21). PEG-40S is also a biocompatible and non-ionic surfactant widely used in pharmaceutical products (22). It can also be used as an emulsifier, solubilizer, surface-modifying element, and in the preparation of lipid-coated microbubbles (23). This novel system has the potential to be used either as scaffolds and implant coatings, or in drug delivery applications that require a large surface area of administration often provided by polymer films and scaffolds (3).

\section{Materials and Methods}

\subsection{Materials and preparation of polymeric solutions}

Polymeric/lipids/surfactant solutions with different concentrations were prepared in the experiments, as shown in table 1. For the solution preparation, sodium alginate powder (SigmaAldrich, Poole, U.K.) was initially dissolved in deionized water followed by the addition of the surfactant polyethylene glycol-40-stearate (PEG-40S, Sigma-Aldrich, Poole, U.K., with a density of $1300 \mathrm{~kg} / \mathrm{m}^{3}$ ) under continuous stirring for 1 hour. In the cases where phospholipids were required, a phospholipid (hydrogenated L- $\alpha$-phosphatidylcholine, Type I-EH, $0.5 \%$ w/w, powder) was added to the mixture and was then further stirred for approximately 4 hours.

\subsection{Characterization of polymeric solutions}

A Brookfield DV-11 Ultra programmable rheometer (Brookfield Engineering Laboratory Inc., Middleboro, MA, U.S.A.) was used to measure the viscosity of alginate solutions. The surface tension was measured using a Kruss tensiometer (model-K9, Kruss GmbH, Germany). To ensure the accuracy of the measurements, seven readings were taken. and the average is reported. All of the measurements were conducted at ambient temperature $\left(22{ }^{\circ} \mathrm{C}\right)$.

\subsection{Preparation of polymeric microbubbles}

The set-up used in this work for T-junction microbubbling is illustrated in figure 1. This microfluidic device was fabricated with poly(methyl methacrylate) (PMMA) using CNC machining. The T-junction device has two perpendicular Teflon capillaries (length of each is $50 \mathrm{~mm}$ ) having the same inner diameter $(200 \mu \mathrm{m})$. The vertical capillary provides the bubbling gas from a gas cylinder that is connected to a digital manometer to adjust the gas pressure according to the requirements. The other horizontal capillary channels provide the polymeric solution flow, and this is fed and controlled using a 20-ml stainless steel syringe (KD Scientific, Holliston, MA, U.S.A.) connected to a syringe pump (PHD 4400, Harvard). 
For microbubble production, the solution is fed at a constant rate using the syringe pump and the gas pressure is increased gradually until it overcomes the surface tension of the solution. Both the polymeric solution and the gas meet at the intersection zone between the two perpendicular capillaries, microbubbles are then produced at this gas/liquid interface. By adjusting the bubbling pressure and the flow rate of the solution simultaneously, monodispersed microbubbles can be formed. The ability to produce monodispersed microbubbles using a T-junction is exemplified in the Supporting Information (SI) video 1. A constant volume $(1 \mathrm{~mL})$ of the solution was used to produce the microbubbles, which were collected on a glass slide at the outlet capillary and observed using optical microscope until the bubbles burst and the resulting structures were obtained. SI video 2 shows the microbubbles on the glass slide after collection.

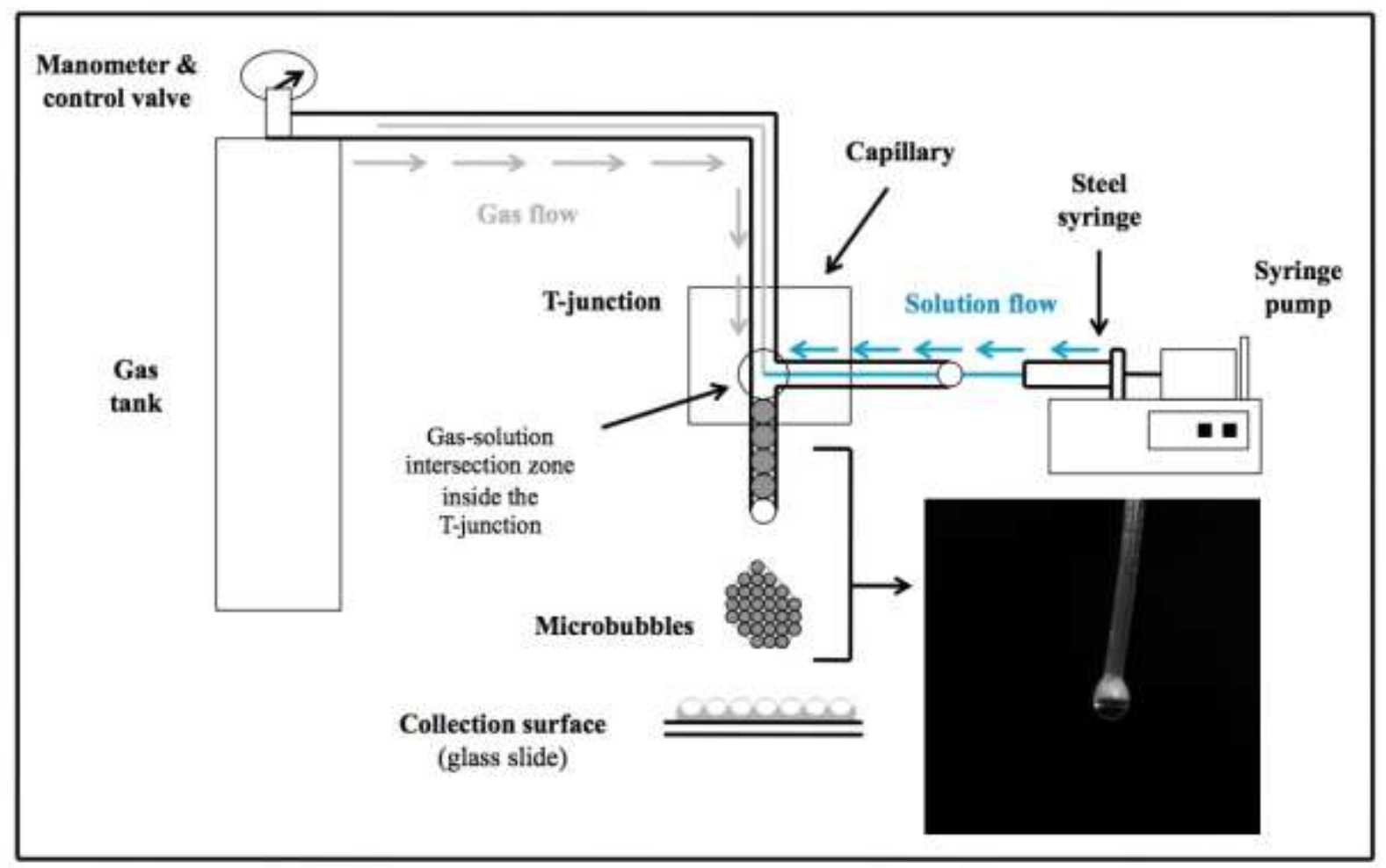

Figure 1: Schematic drawing showing the process of obtaining polymeric microbubbles using the T-junction.

\subsection{Microstructure characterization}

For the examination of microbubbles, an optical microscope (Zeiss Axiotech) fitted with a Nikon Eclipse ME 600 camera was used. The porous structures/films produced by controlling the bursting of various microbubbles were examined using a JEOL JSM-6301F field emission scanning electron microscope (FE-SEM, JEOL Ltd., Herts, U.K.). The samples were sputter 
coated with gold for 2 minutes (Edwards Sputter coater S150B) before the SEM examination, which was at an accelerating voltage of $3-15 \mathrm{kV}$, a working distance of $1-20 \mathrm{~mm}$. The size distribution of the microbubbles, the pore sizes of films produced, and the size of submicrometre particles were determined using the UTHSCSA Image Tool software program (Version 3.00).

\section{Results \& Discussion}

\subsection{The Properties of polymeric solutions}

Table 1: The viscosity and surface tension of the alginate/PEG-40S and alginate/lipid/PEG$40 \mathrm{~S}$ solutions used for microbubbling

\begin{tabular}{|c|c|c|c|c|c|}
\hline Solution & $\begin{array}{c}\text { Alginate } \\
\text { concentration } \\
(\mathrm{wt} \%)\end{array}$ & $\begin{array}{c}\text { Lipid } \\
\text { concentration } \\
(\mathrm{wt} \%)\end{array}$ & $\begin{array}{c}\text { PEG-40S } \\
\text { concentration } \\
(\mathrm{wt} \%)\end{array}$ & $\begin{array}{c}\text { Viscosity } \\
(\mathrm{mPa})\end{array}$ & $\begin{array}{c}\text { Surface tension } \\
(\mathrm{mN} / \mathrm{m})\end{array}$ \\
\hline 1 & 0.1 & & 0.05 & $5.5 \pm 0.7$ & $40.5 \pm 0.8$ \\
\hline 2 & 0.2 & & 0.05 & $7.3 \pm 0.4$ & $42.2 \pm 0.5$ \\
\hline 3 & 0.3 & & 0.15 & $9.5 \pm 0.5$ & $38.5 \pm 0.5$ \\
\hline 4 & 0.5 & & 0.25 & $13.4 \pm 0.5$ & $37.4 \pm 0.7$ \\
\hline 5 & 1 & 0.25 & 0.25 & $18.6 \pm 0.4$ & $38.9 \pm 0.3$ \\
\hline 6 & 1 & 0.25 & 0.5 & $21.5 \pm 0.6$ & $35.4 \pm 0.5$ \\
\hline 7 & 1 & 0.25 & 0.75 & $22.5 \pm 0.8$ & $33.5 \pm 0.7$ \\
\hline 8 & 1 & & & & \\
\hline
\end{tabular}

The viscosity and surface tension of the different solutions are known to have an effect upon microbubbling $(14,24)$. Increasing the polymer concentration was found to increase the viscosity of the solutions in all cases, as shown in Table 1 . The viscosity of alginate solutions is dependent on the concentration of alginate solution (25). An ability to control the viscosity of the solution is crucial in this work, because solutions with higher viscosities were found to suppress the bubble bursting process (17). Furthermore, it has been found that the surface tension of the solutions decreased with an increasing surfactant concentration when the polymer concentration was kept constant. The surface tension of the bubble film (shell) is another critical factor for consideration because bubbles having a higher surface tension are characterized by faster film drainage (26). This important variable will be discussed in the following section.

\subsection{T-junction Microbubbling}


Our choice of the T-junction method was based on its ability to produce monodisperse microbubbles using two main processing variables: the bubbling pressure and the flow rate. To minimize the effect of the shear forces on the solutions flowing within the capillaries for a given microbubble size to be produced, we intended to use the lowest possible flow rate (60 to $115 \mu \mathrm{l} / \mathrm{min}$ ), relatively shorter microfluidic capillaries $(50 \mathrm{~mm})$ and larger capillary diameters $(200 \mu \mathrm{m})$. Increasing the flow rate, decreasing the capillary diameter, or increasing the length of the microfluidic channels would increase the velocity of the flow and increase the intensity of the sheer forces to which the solution is subjected (27). This can affect the physiochemical properties of the solutions, especially in the case of viscoelastic sheer thinning solutions, such as alginate solutions (21). For a specific gas pressure, a maximum flow rate can be used before the solution flow overcomes the gas flow, leading to the inability to produce microbubbles (13). Furthermore, viscosity of the solutions was measured at the conditions before and after processing, and it was found that processing had almost no effect on the viscosity of the solutions. Therefore, it was assumed that no significant effect on the mechanisms of producing porous films in the current study would occur as a result of the dynamics of the process.

After collection on a flat dry surface (glass slide), the monodispersed microbubbles were found to align themselves in an ordered pattern, in contrast to polydisperse microbubbles. Because of their similar sizes, microbubbles spontaneously assembled in the form of organized ordered lattices, called microfluidic crystals (28), which, if retained, can help in the formation of the intended regular porous structures, and this will be discussed in section 3.4.

\subsection{Effect of the surfactant concentration on the porous structures}

To investigate the effect of the surfactant on the porous structures, we initially produce microbubbles from a fixed concentration of a hydrophilic alginate polymer and a series of surfactant (PEG-40S) concentrations, starting from a relatively low percentage (0.05 wt\%). Surfactants can control the stability of bubbles before they burst, and the duration of stability can be used as an indication of the bubble shell thickness at the bursting point (29). The bubble shell often has its minimum thickness at the centre and then this continuous decrease in thickness progresses to a larger area of the shell as the film drainage continues (30). Therefore, it is expected that accelerating or inhibiting the bubble shell drainage could also affect the bubble diameter at bursting, which would, in turn, control the pore size that bubbles leave behind within the polymeric structure after they burst. It was found that microbubbles having 
a PEG-40S concentration of $0.05 \mathrm{wt} \%$ are unable to produce porous structures, because they lose their arrangement/structure completely after a short period. Despite being initially monodispersed and stable (for the first few minutes), the microbubbles went through rapid drainage, as shown in figure 2, also exemplified in SI video 3, then completely lost their initial spherical structure and arrangement, leaving behind threads of the polymeric material.

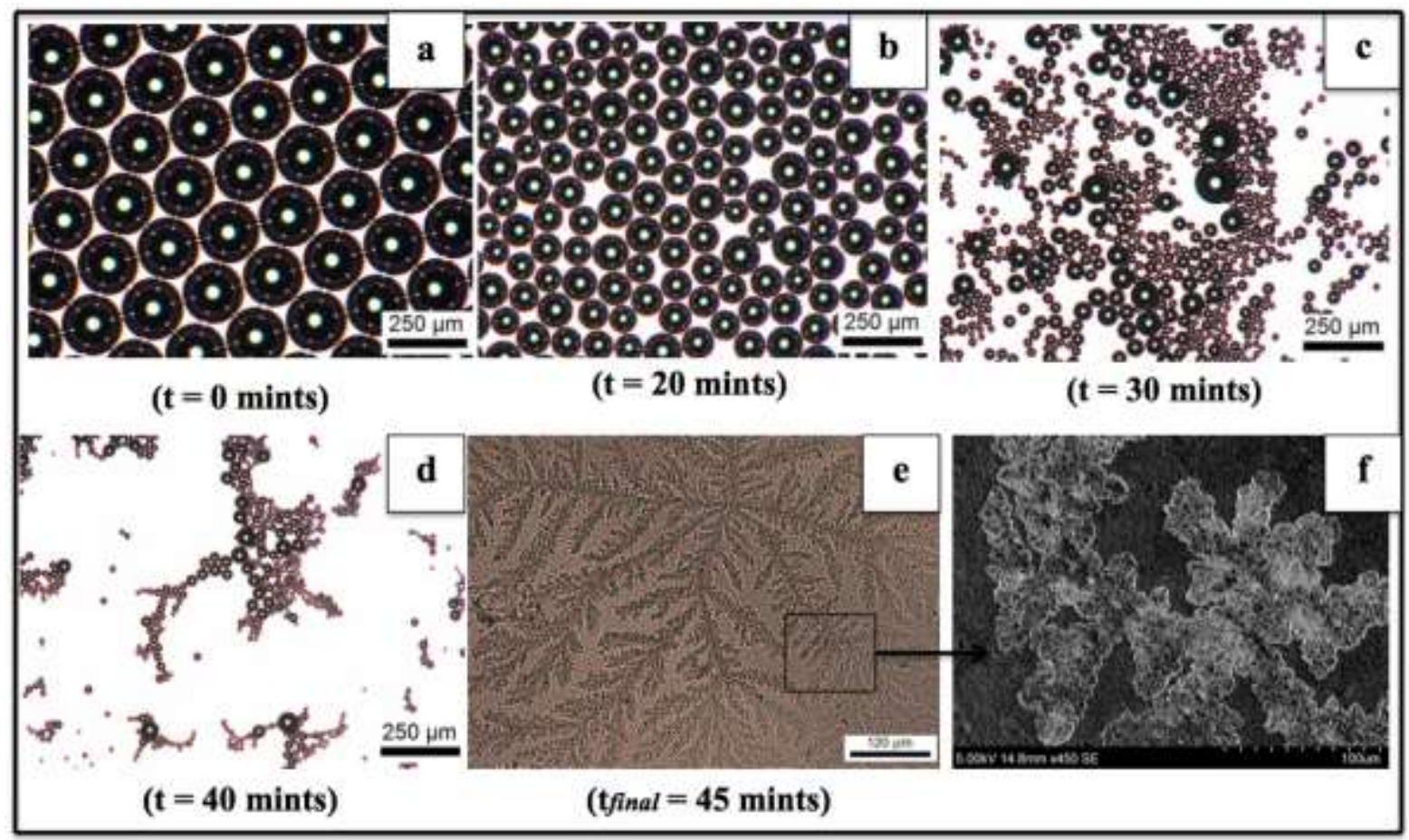

Figure 2: Optical micrographs showing the size changes of alginate/PEG-40S microbubbles made from a low surfactant concentration $(0.05 \mathrm{wt} \%$, sol. $2 /$ table 1$)$ at times of (a) $0 \mathrm{~min}$, (b) $20 \mathrm{~min}$, (c) $30 \mathrm{~min}$, (d) $40 \mathrm{~min}$ and (e) $45 \mathrm{~min}$. The size of microbubbles decreased with time and the spherical structure was completely lost after 45 mins. (f) Scanning electron micrograph showing a branched-like residual structure of the microbubbles in the absence of a bursting process.

The size of monodisperse microbubbles can be controlled by the flow rate of the solution and the bubbling pressure, as demonstrated previously (17). At a constant flow rate of $220 \mu \mathrm{l} / \mathrm{min}$, it was found that the average microbubble size increased from approximately 70 to $150 \mu \mathrm{m}$, when the bubbling pressure was increased from 95 to $165 \mathrm{kPa}$. The rapidly shrinking microbubbles started forming branches of small microbubbles, which eventually left threads of polymeric material in similar branched positions. The mobility of the boundaries between microbubbles can be caused by the rapid drainage of the shell of the bubbles when low amounts of surfactants are used (26), and a similar finding was observed in the alginate system. This is a characteristic of microbubbles when the surfactant concentration was low. 
As shown in figure 3, it took around 45 minutes for the largest microbubbles $(150 \mu \mathrm{m})$ to completely lose their spherical structure, compared to around 25 minutes for the smallest microbubbles $(70 \mu \mathrm{m})$. This shows that at a low surfactant concentration, the bubble diameter was less dominant in determining the bursting of microbubbles, because microbubbles in figure $2(\mathbf{a}, \mathbf{b}, \mathbf{c})$ did not burst.

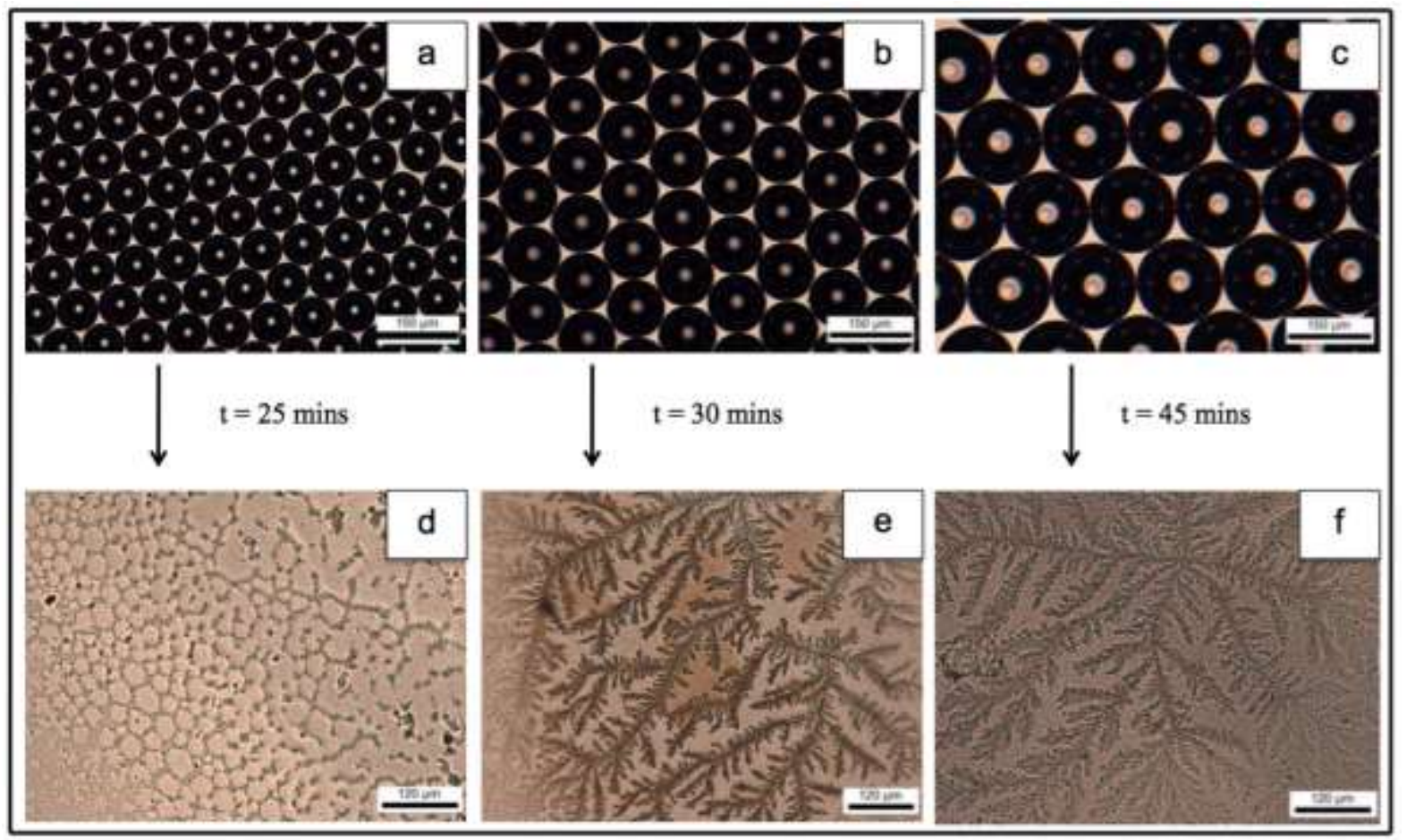

Figure 3: Optical micrographs showing (a) $\sim 70 \mu \mathrm{m}$, (b) $\sim 100 \mu \mathrm{m}$ and (c) $\sim 150 \mu \mathrm{m}$ alginate/PEG-40S (from sol.2 in Table 1) microbubbles immediately after production, and after the process of shell thinning and complete drying at times of (d) 25 mins, (e) 30 mins and (f) 45 mins for the three corresponding microbubble groups respectively.

The size of microbubbles was found to be directly proportional to the duration of stability of microbubbles before their eventual bursting. The stability of bubbles is a known function of their radius, which governs the Laplace pressure, but many other factors can affect stability, e.g. the packing in the shell, shell impurities. Our observation agrees with previous studies that in general larger microbubbles have greater stability compared to smaller ones. Filho et al. (31) found that microbubbles with an average diameter of $300 \mu \mathrm{m}$ were $170 \%$ more stable compared to microbubbles with an average diameter of $70 \mu \mathrm{m}$.

To overcome the obstacle of microbubbles completely losing their alignment and shape to form the backbone of the intended porous structure, microbubbles from alginate/surfactant solutions 
with a weight ratio of 2:1 were used. A larger alginate/surfactant ratio was found to suppress the bursting of microbubbles and produce capsule-like structures, as shown in figure 4). The pore size was ranging from 2 to $17 \mu \mathrm{m}$, smaller in comparison to larger and more regular pores shown in figure 5. Additionally, increasing the surfactant/polymer concentration can be undesirable when the polymer is expected to form the backbone of the structure, such as in the case of alginate after cross-linking. Therefore, the surfactant concentration in our study was kept as low as possible.

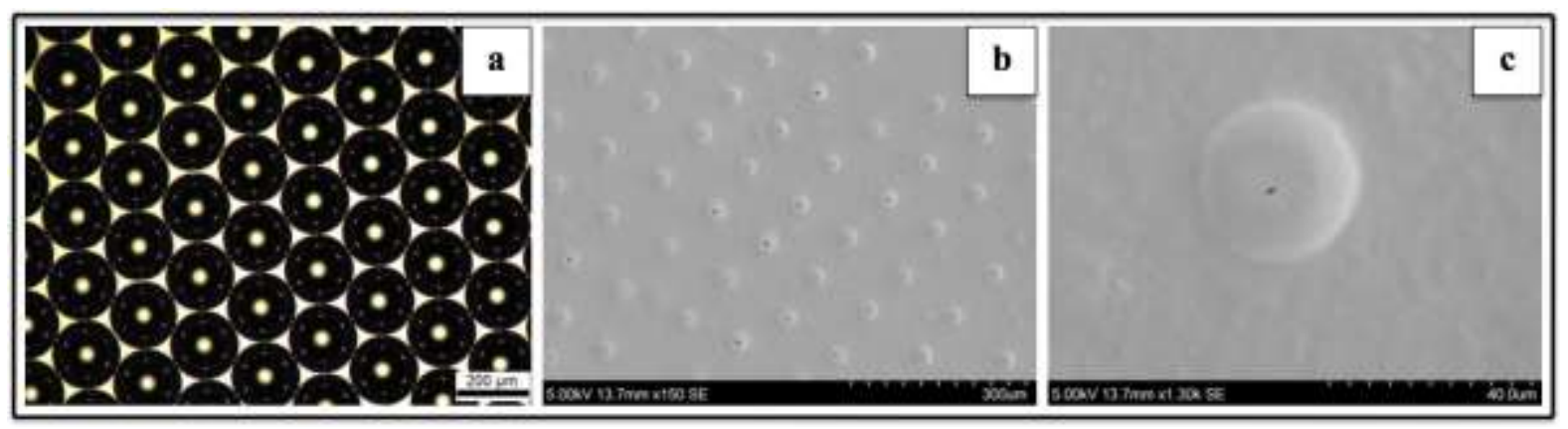

Figure 4: (a) Optical micrograph showing microbubbles prepared from alginate/PEG-40S (sol. 5/Table 1) having a high alginate concentration $(1 \mathrm{wt} \%)$ and a relatively low concentration of PEG-40S (0.25 wt\%) just after preparation, (b) Scanning electron micrograph showing the resulting structure formed at the end of thinning process of the microbubbles after $35 \mathrm{mins}$, (c) Higher magnification scanning electron micrograph showing the capsule-like structure that microbubbles turned into at the end of the thinning process.

\subsection{Effect of the polymer concentration on the porous structures:}

The bubble formation and burst were investigated when the alginate concentration was varied from 0.1 to $0.5 \mathrm{wt} \%$ and the PEG-40S concentration was varied from 0.05 to $0.25 \mathrm{wt} \%$. The three groups of monodisperse microbubbles were produced with a similar range of as prepared sizes (120 to $140 \mu \mathrm{m})$. The microbubbles maintained their alignment and durations of stability to various degrees before the structures shown in figure 5 were formed. Microbubbles from solution with the least amount of alginate and surfactant as well as the lowest viscosity went through the shortest duration of stability of 56 mins, in comparison with 67 and 75 mins, respectively, for the microbubbles made from 0.3 and $0.5 \mathrm{wt} \%$ alginate solution. The size and shape of pores were also varied, as shown in figure 5. As expected, the strut thickness increased with the alginate concentrations increasing from 0.1 to $0.5 \mathrm{wt} \%$. This is reflected in the variation of resulting pore sizes of the films, as shown in figure 6. 


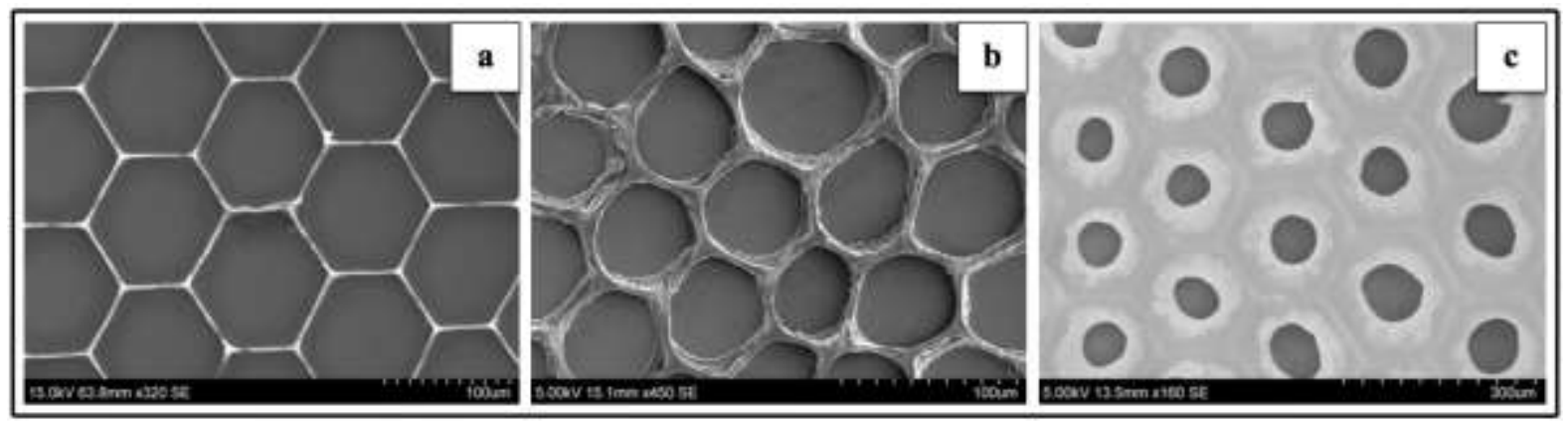

Figure 5: Scanning electron micrographs showing the relics obtained from the bursting of alginate microbubbles (sol. 1, 3 and 4/table 1), having three polymer/PEG-40S concentrations with a fixed ratio of 2:1, where the polymer (alginate) concentrations increased from (a) 0.1 wt $\%$, (b) $0.3 \mathrm{wt} \%$ to (c) $0.5 \mathrm{wt} \%$.

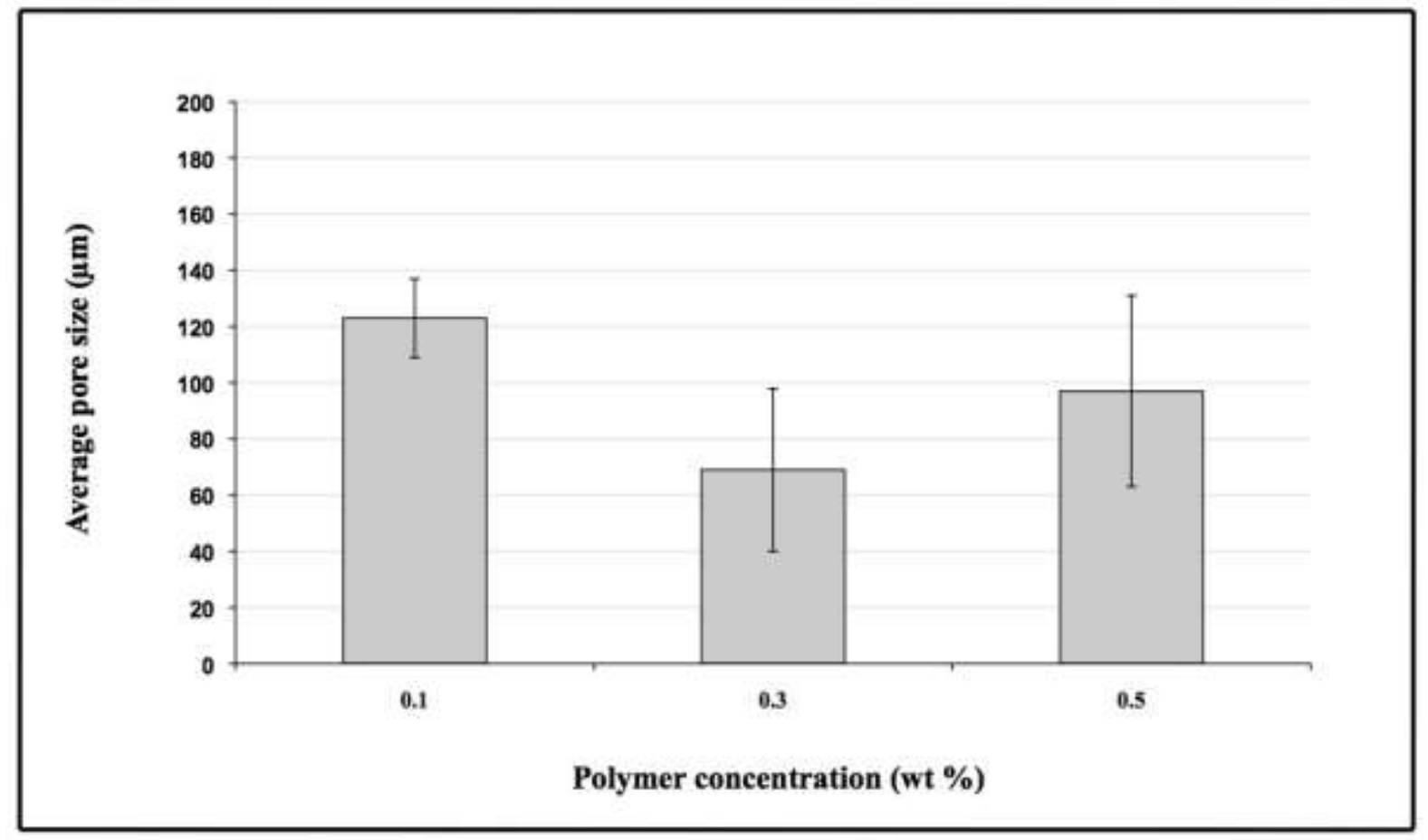

Figure 6: Comparison of the average pore sizes of porous films obtained from microbubbling of three solutions with a fixed polymer/PEG-40S concentration ratio of $2: 1$, where alginate concentrations varied from $0.1,0.3$ to $0.5 \mathrm{wt} \%$.

It is expected that the longer stability provided an extended duration for the bubble shell thinning to occur, because coating the microbubbles with PEG-40S has been found to significantly enhance the stability of bubbles (32). Increasing the viscosity of the bubble shell is known to extend the thinning process (33). However, the increase in the duration of the thinning process can lead to, in some cases, a relatively unsymmetrical deposition of the material, and thus a variation in the pore size and a decrease in the average pore size in the 
films when compared to the initial microbubble size for obtaining the films, as shown in figure 6. The average pore size of the film produced from $0.1 \mathrm{wt} \%$ alginate solution was $122 \pm 15 \mu \mathrm{m}$, while the average pore size was $70 \pm 30 \mu \mathrm{m}$ made from $0.3 \mathrm{wt} \%$ alginate solution. Not only it has a higher standard deviation but also a greater shrinkage (around 40\%) from the original microbubbles size $(\sim 120 \mu \mathrm{m})$. In contrast, microbubbles from the highest polymer $(0.5 \mathrm{wt} \%)$ and surfactant concentrations showed the greatest duration of stability, and less noticeable reduction in the pore size obtained, which might be a result of the greater material concentrations within the bubble shell that can induce a more uniform shrinkage and material deposition within the boundaries between microbubbles (plateau borders).

\subsection{Effect of surfactant on the porous structures:}

The surfactant is known to play a dual role in the process of film formation. The first is stabilizing the microbubbles through controlling the diffusion of gas from the bubbles, preventing the coalescence of adjacent microbubbles (34) and losing their initial alignment, to ultimately produce films with nearly uniform structure and pore size. The second is controlling/extending the thinning process of the bubbles (35) that allows the gradual accumulation of the polymeric material between the voids over an extended duration of time, leading to producing films with smooth surfaces. Therefore, there might be a trade-off between extending the microbubble thinning duration and the variation in the features of the films. Furthermore, microbubbles from higher alginate concentrations produced structures with larger dimensions between pores, which is probably due to the greater amount of the polymeric material within the bubble shell, resulting from the higher polymer and surfactant concentrations used.

It was noted that the porous films assumed a near-polyhedral shape in all cases, especially at the lowest material (polymer and surfactant) concentrations (figure 5 a and b). This can be caused by the lower liquid volume fraction within the produced cluster of microbubbles, initiated by the relatively low liquid/gas flow ratios used to obtain the microbubbles, thus reducing shear stresses on the processed solution. Polyhedral porous structures have been previously referred to as 'dry foam' and can be generated by microfluidic methods using relatively lower liquid to gas fractions, in contrast to wet foams, which are formed at a higher liquid to gas fractions and are characterized by a more spherical shape (36). Producing clusters of microbubbles with lower liquid to gas factions is desirable for porous film production 
because it minimize the amount of liquid or material that can suppress the bursting, otherwise leading to irregular features in the films.

It was showed that, by adjusting the polymer/surfactant ratio, the ability to control the thinning of microbubbles can be predicted, which could lead to producing porous structures with controlled features. However, when higher polymer concentrations (more than $0.5 \mathrm{wt} \%$ ) are used for desirable mechanical properties and for tuning the encapsulation efficiency and degradation profile of the resulting products, it can be challenging because increasing the polymer concentration can inhibit bursting (17), and result in structures lacking the intended defined/large pore shape, as shown in figure 4. Therefore, phospholipids were added to the polymeric systems as a result of their well-established ability to self-assemble around the inner gaseous gas core of the microbubble, providing a stronger (i.e., more elastic) shell, which makes the bubbles more suitable for the addition to other functional entities (37), and also allows for the incorporation of higher polymer concentrations.

The hypothesis is that, by achieving a longer stability and extended bubble shell thinning, the effective bursting of the bubble shell will still be promoted, which will eventually produce uniform porous structures. One of the most crucial functioning elements within the bubble shell is the surfactant as a result of its ability to create a barrier for the gas transfer in and out of the bubble shell (38). The non-uniform distribution of the surfactant within the bubble shell can also result in a difference in the surface tension (surface tension gradient) across the bubble film (39) as a result of Marangoni effect, thus causing slower or accelerated thinning according to the direction of the surface tension gradient across the bubble film (40). As for the chosen polymeric system (alginate + lipids + PEG-40S solution), PEG-40S has also been known to act as an emulsifier, capable of improving the lipid dispersion and preventing the coalescence of the microbubbles (37). Preventing the coalescence of bubbles is crucial to limit the production of porous structures with a polydisperse pore size. Therefore, increasing the concentration of the surfactant further seemed potentially useful. The effect of the emulsifier was demonstrated when three alginate solutions having fixed alginate and lipid concentrations of 1 and $0.25 \mathrm{wt} \%$, respectively, and three different concentrations of PEG-40S $(0.25,0.5$ and $0.75 \mathrm{wt} \%)$ were investigated. The first two groups of alginate microbubbles produced porous structures with irregular-shaped pores, as shown in figure $\mathbf{7 a}$ and figure $\mathbf{7 b}$. The formation of porous films with an irregular pore shape is exemplified in SI video 4. However, alginate microbubbles with 
the highest PEG-40S concentration $(0.75 \mathrm{wt} \%)$ resulted in a porous structure with nearspherical pores, as shown in figure 7c and in examples discussed later. This can be the result from a better dispersion of the material within the bubble shell and a thinner shell thickness at the bursting moment, which lead to efficient bursting and more 'open' pores, as shown in figure 7c, compared to the irregular-shaped and 'closed' pores, as shown in figure 7a.

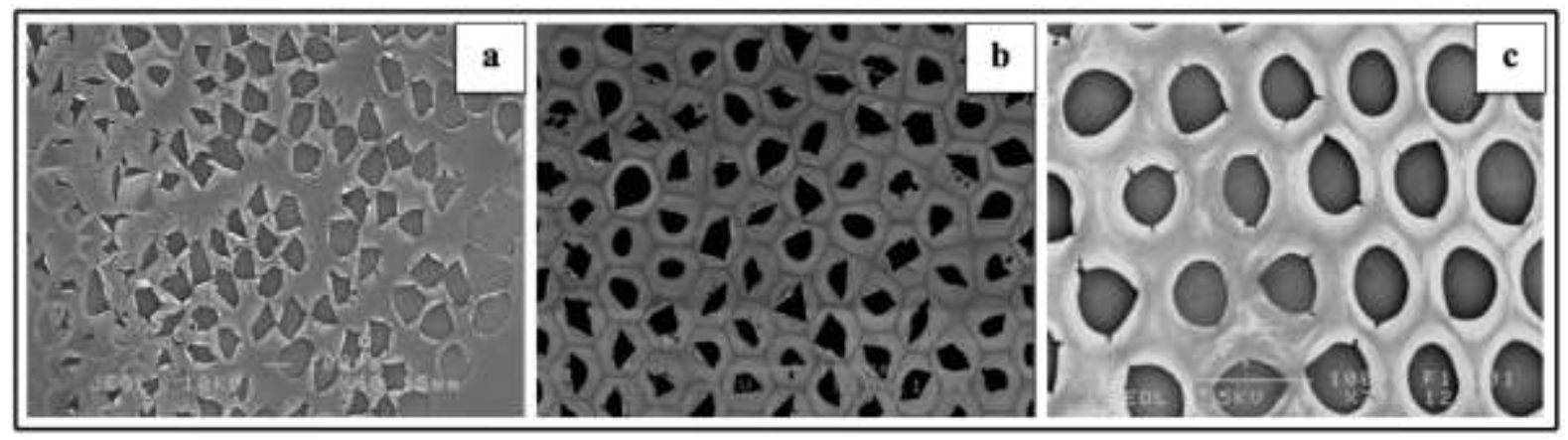

Figure 7: Scanning electron micrographs showing the porous structures resulting from the bursting of alginate/lipid/PEG-40S microbubbles (sol. 6, 7, 8/table 1), having constant alginate ( $1 \mathrm{wt} \%)$ and phospholipid (0.25 wt \%) concentrations, and three PEG-40S concentrations of (a) $0.25 \mathrm{wt} \%$, (b) $0.5 \mathrm{wt} \%$ and (c) $0.75 \mathrm{wt} \%$.

It is important to note that microbubbles prepared from the same alginate/surfactant concentrations but with/without lipids (solutions 5 and 6/Table 1) went through different shrinkage and bursting mechanisms. Microbubbles that did not contain lipids went through considerable shrinkage and ended up bursting through to contain a 'narrow', few micrometers in size and voids on their surface, as shown in figure 4. On the other hand, microbubbles containing $0.25 \mathrm{wt} \%$ lipids went through a relatively lower shrinkage in microbubble size and produced more 'open' pores, despite having irregular shapes. This shows the important role of lipids in inducing an efficient bursting process as a result of the more 'elastic' nature of lipidbased microbubbles (37). The formation of porous films with a more uniform pore shape is exemplified in SI video 5. Furthermore, microbubbles from this solution had the longest lifetime before bursting (approximately 90 mins). The extended lifetime can be crucial in defining the structures resulting from bubble bursting, because bubbles are considered to have strong time-dependent properties (41). Microbubbles having the highest surfactant concentration also maintained their monodispersity and alignment to a considerable extent throughout the thinning process, just before the bursting of microbubbles, as shown in figure $\mathbf{8 b}$, until the resulting structure was formed, as shown in figure 8c. 


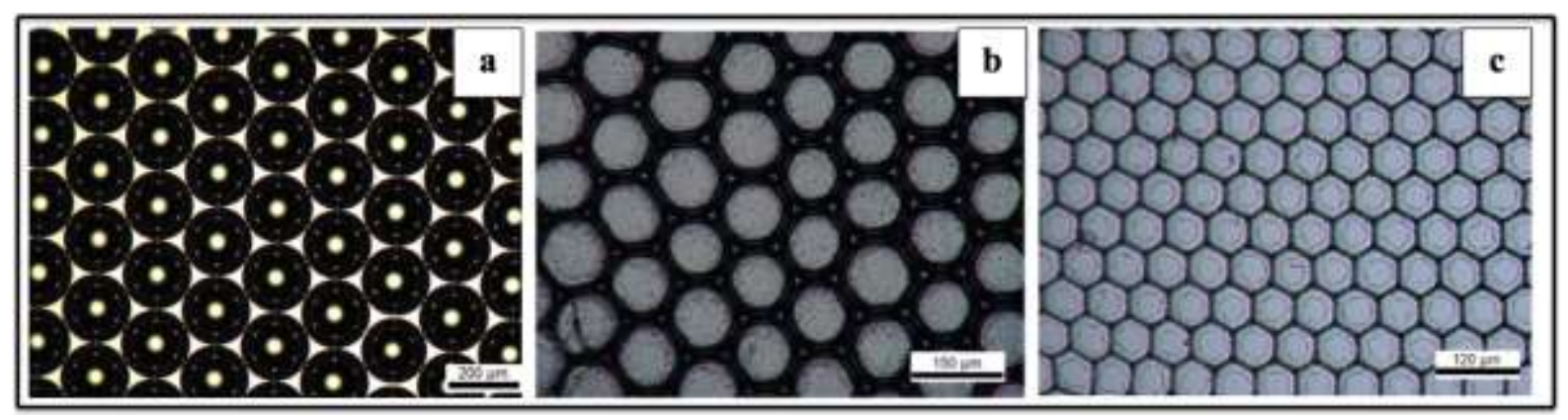

Figure 8: Optical micrographs showing the alginate/lipid/PEG-40S microbubbles (sol.8 in Table 1) at the time points of (a) just after preparation (b) just before bursting, and (c) after bursting.

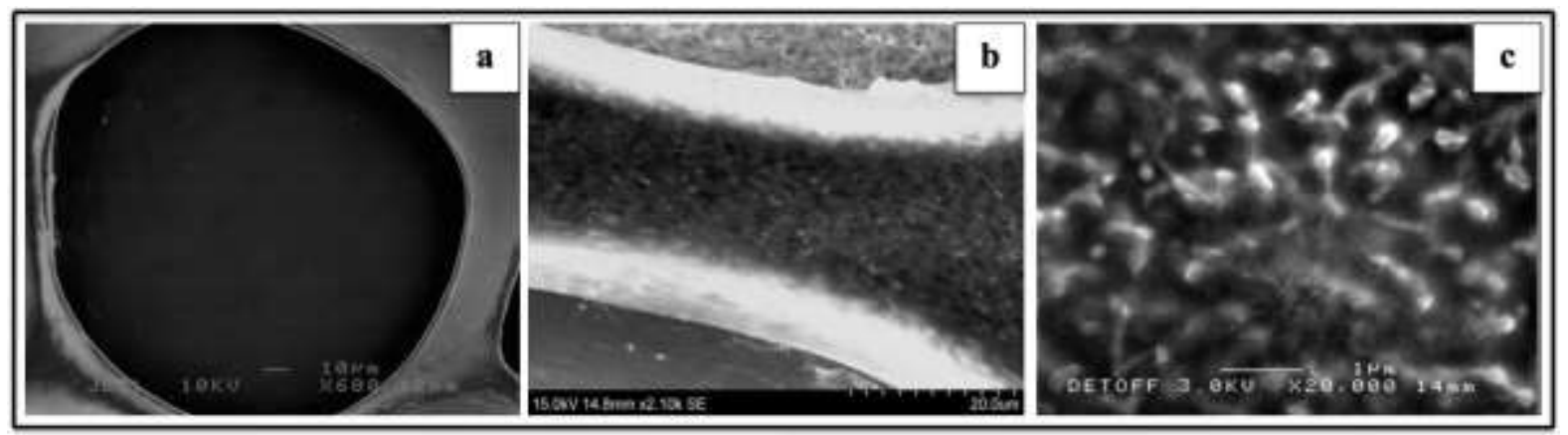

Figure 9: Scanning electron micrographs showing the (a) the structure of the pore obtained at the highest PEG-40S concentration (sol. 8 in Table 1) (b) lower magnification image showing nano-patterned surface (c) embedded nanoparticles produced as a result of the efficient bursting of the microbubbles.

The efficient bursting process from this group of microbubbles resulted in a nano-patterned surface, caused by the nano-droplets produced during the bursting that became embedded in the porous film, as shown in figure 9c. Previous reports (42) showed that as the bubble cap reaches a critical thickness, the shell breaks into fine droplets with a different size range, starting from a sub-micrometre range. This notion is similar to our findings as particles with an average diameter of $315 \mathrm{~nm}$ were found embedded within the surface of the porous film, as shown in figure 9 a and $\mathbf{b}$. The ability to control the surface structure of a porous film is very important, as the surface properties have impacts on the cellular functionalities, such as cellular adhesion, proliferation, differentiation etc. (43).

Our results show that, through controlling the initial size of microbubbles using a microfluidic method, such as a T-junction, and adjusting the solution properties (i.e., concentrations of polymer, surfactant and emulsifier), we are able to produce well-defined porous structures with a nano-patterned surface. The description of this novel preparation method is shown in figure 
10. We have advanced from controlling the bubbles thinning and bursting (44) to establishing a new processing method with the capability to achieve not only a well-defined porous structure but also a regulated strut morphology. To the best of our knowledge, this is the first time such a well-defined porous structure has been produced using a microfluidic processing without additional post processing treatment.

\section{Conclusions:}

Highly monodispersed polymeric microbubbles were produced to prepare porous films. Biocompatible polymeric solutions were used, and film structures with nearly uniform pore size and shape were obtained. The thinning process of the microbubble can be controlled using the aid of surfactants and lipids, leading to the formation of porous structures with spherical shapes, by promoting an efficient bursting process. Additionally, higher polymer concentrations can result in suppressing the bursting process and lead to irregular and sharpedged pores, but this can be overcome by varying the concentration of the emulsifier to lead to the formation of uniform, spherical pore structures. Finally, the porous films produced can be tailored to have a nano-patterned strut surface, by promoting the efficient production of droplets from the bursting of microbubbles. This novel processing and forming will offer a new

platform to further tailor the structure and properties of porous polymeric films for further functional applications. 


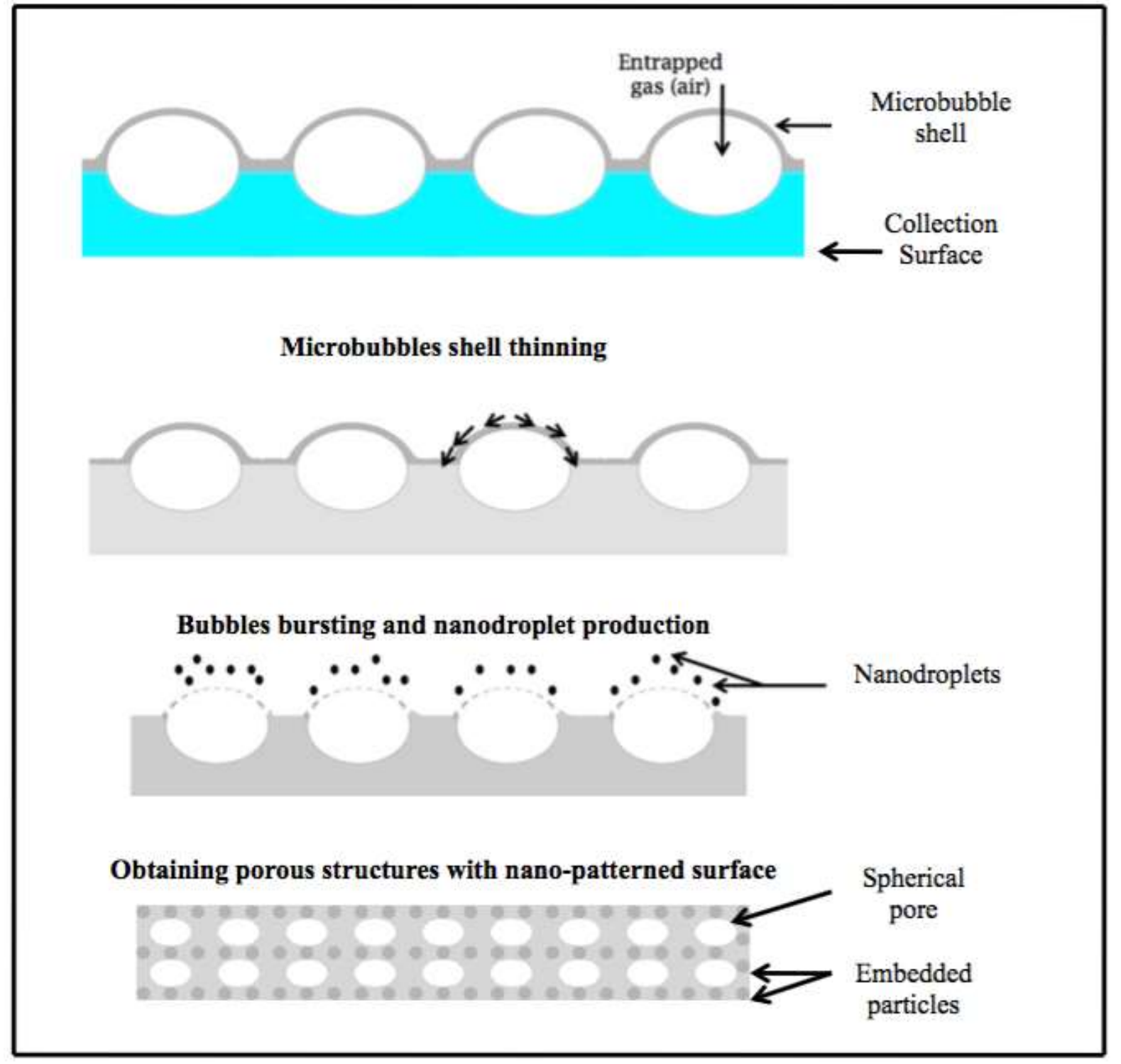

Figure 10: Schematic drawing showing the process of obtaining porous structures (films and scaffolds) from the bursting of microbubbles. 


\section{References}

1. Arai, K.; Tanaka, M.; Yamamoto, S.; Shimomura, M. Effect of Pore Size of Honeycomb Films on the Morphology, Adhesion and Cytoskeletal Organization of Cardiac Myocytes. Colloids and Surfaces A: Physicochem. Eng. Aspects. 2008, 313$314,530-535$.

2. Cai, Y.; Newby, B. Porous Polymer Films Templated by Marangoni Flow-Induced Water Droplet Arrays. Langmuir. 2009, 25 (13), 7638-7645.

3. Pereira, G.; Guterres, S.; Balducci, A.; Colombo, P.; Sonvico, F. Polymeric Films Loaded with Vitamin E and Aloe vera for Topical Application in the Treatment of Burn Wounds. BioMed. Res. Int. 2014, 641590.

4. Kim, B.; Zhang, X.; Borteh, H.; Li, Z.; Guan, J.; Zhao, Y. Fabrication of Porous Microtent Structures Toward an in vitro Endothelium Model. J. Micromech. Microeng. 2012, 22, 085001.

5. Chung, K.; Mishra, N.; Wang, C.; Lin, F.; Lin, K. Fabricating Scaffolds by Microfluidics. Biomicrofluidics 3. 2009, 022403.

6. Zmora, S.; Glicklis, R.; Cohen, S. Tailoring the Pore Architecture in 3-D Alginate Scaffolds by Controlling the Freezing Regime During Fabrication. Biomaterials. 2002, 23, 4087-4094.

7. Parhizkar, M.; Stride, E.; Edirisinghe, M. Preparation of Monodisperse Microbubbles Using an Integrated Embedded Capillary T-junction with Electrohydrodynamic Focusing. Lab on a Chip. 2014, 14 (14), 2437-2446.

8. Widawski, G.; Rawiso, M.; Francois, B. Self-Organized Honeycomb Morphology of Star Polymer Polystyrene Films. Nature. 1994, 369, 387-389.

9. Zhang, W.; Wan, L.; Meng, X.; Li, J.; Ke, B.; Chen, P.; Xu, Z. Macroporous, ProteinContaining Films Cast from Water-in-Oil Emulsions Featuring a Block-Copolymer. Soft Matter. 2011, 7, 4221.

10. Wang, C.; Yang, K.; Lin, K.; Wu, C.; Liu, Y.; Lin, F.; Chen, I. A Biomimetic Honeycomb-Like Scaffold Prepared by Flow-Focusing Technology for Cartilage Regeneration. Biotechnology and Bioengineering. 2014, 111 (11), 2338-2348.

11. Gomes, M.; Reis, R. Biodegradable Polymers and Composite in Biomedical Applications: from Catgut to Tissue Engineering. Int. Mater. Rev. 2004, 49 (5), 261273.

12. Parhizkar, M.; Sofokleous, P.; Stride, E.; Edirisinghe, M. Novel Preparation of Controlled Porosity Particle/Fibre Loaded Scaffolds Using a Hybrid Micro-fluidic and Electrohydrodynamic Technique. Biofabrication. 2014, 6, 045010. 
13. Pancholi, K.; Stride, E.; Edirisinghe, M. Dynamics of Bubble Formation in Highly Viscous Liquids. J. Drug Target. 2008, 24, 4388-4393.

14. Parhizkar, M.; Edirisinghe, M.; Stride, E. Effect of Operating Conditions and Liquid Physical Properties on the Size of Monodisperse Microbubbles Produced in a Capillary Embedded T-junction Device. Microfluid Nanofluid. 2013, 14, 797-808.

15. Ekemen, Z.; Ahmed, Z.; Stride, E.; Kaplan, D.; Edirisinghe, M. Electrohydrodynamic Bubbling: An Alternative Route to Fabricate Porous Structures of Silk Fibroin Based Materials. Biomacromolecules. 2013, 14, 1412-1422.

16. Russell, L.; Singh, E. Submicron Salt Particle Production in Bubble Bursting. Aerosol Sci. Technol. 2006, 40, 664-671.

17. Elsayed M.; Huang, J.; Edirisinghe, M. Bioinspired Preparation of Alginate Nanoparticles Using Microbubble Bursting. Materials Science and Engineering $C$. 2015, 46, 132-139.

18. Ng, R.; Zang, R.; Yang, K.; Liu, N.; Yang, S. Three-dimensional Fibrous Scaffolds with Microstructures and Nanotextures for Tissue Engineering. RSC Advances. 2012, 2, 10110-10124.

19. Malafaya, P.; Silva, G.; Reis, R. Natural-Origin Polymers as Carriers and Scaffolds for Biomolecules and Cell Delivery in Tissue Engineering Applications. Adv. Drug Deliv. Rev. 2007, 59, 207-233.

20. Goh, C.; Heng, P.; Huang, E.; Li, B.; Chan, L. Interactions of Antimicrobial Compounds with Cross-linking Agents of Alginate Dressings. Journal of Antimicrobial Chemotherapy. 2008, 62, 105-108.

21. Patra, S.; Bal, D.; Ganguly, S. Diffusion of Moisture from Hydrogel Scaffold with Induced Porosity from Self-Assembled Bubbles. Drying Technology. 2015, 33, 336345.

22. Jiao, J. Polyoxyethylated Nonionic Surfactants and their Applications in Topical Ocular Drug Delivery. Adv. Drug Deliv. Rev. 2008, 60, 1663-1673.

23. Ahmed, B.; Stride, E.; Edirisinghe, M. Calcium Alginate Foams Prepared by a Microfluidic T-Junction System: Stability and Food Applications. Food Bioprocess Technol. 2012, 5 (7), 2848-2857.

24. Parhizkar, M.; Edirisinghe, M.; Stride, E. The Effect of Surfactant Type and Concentration on the Size and Stability of Microbubbles Produced in a Capillary Embedded T-junction Device. RSC Adv. 2015, 5, 10751. 
25. Herrero, E.; Martin Del Valle, E.; Galan, M. Development of a New Technology for the Production of Microcapsules Based in Atomization Processes. Chem. Eng. J. 2006, $117,137-142$.

26. Kocárkova, H.; Rouyer, F.; Pigeonneau, F. Film Drainage of Viscous Liquid on Top of Bare Bubble: Influence of the Bond number. Physics of Fluids. 2013, 25, 022105.

27. Hallow, D.; Seeger, R.; Kamaev, P.; Prado, G.; LaPlaca, M.; Prausnitz, M. ShearInduced Intracellular Loading of Cells With Molecules by Controlled Microfluidics. Biotechnology \& Bioengineering. 2008, 99, 846-854.

28. Raven, J.; Marmottant, P. Microfluidic Crystals: Dynamic Interplay between Rearrangement Waves and Flow. Physical Review Letters. 2009, 102, 084501.

29. Lhuissier, H.; Villermaux, E. Bursting Bubble Aerosols. J. Fluid Mech. 2012, 696, 544.

30. Li, D. Coalescence Between Small Bubbles: Effects of Surface Tension Gradient and Surface Viscosities. J. Colloid and Interface Sci. 1996, 181 (1), 34-44.

31. Filho, W.; Schneider, F.; Morales, R. Evaluation of Stability and Size Distribution of Sunflower Oil-Coated Micro Bubbles for Localized Drug Delivery. Biomed. Eng. Online. 2012, 11, 71.

32. Lee, M.; Lee, D. Elastic Instability of Polymer-Shelled Bubbles Formed From Air-inOil-in-Water Compound Bubbles. Soft Matter. 2010, 6, 4326-4330.

33. Allan, R.; Charles, G.; Mason, S. The Approach Of Gas Bubbles To A Gas/Liquid Interface. J Colloid Science. 1961, 16, 150-165.

34. Huerre, A.; Miralles, V.; Jullien, M. Bubbles and Foams in Microfluidics. Soft Matter. 2014, 10, 6888-6902.

35. Hahn, P.; Chen, J.; Slattery, J. Effects of London-van der Waals Forces on the Thinning and Rupture of a Dimpled Liquid Film as a Small Drop or Bubble Approaches a FluidFluid Interface. AlChE Journal. 1985, 31, 12.

36. Marmottant, P.; Raven, J. Microfluidics With Foams. Soft Matter. 2009, 5, 3385-3388.

37. Borden, M.; Longo, M. Dissolution Behavior of Lipid Monolayer-Coated, Air-Filled Microbubbles: Effect of Lipid Hydrophobic Chain Length. Langmuir. 2002, 18, $9225-$ 9233.

38. Mohamedi, G.; Azmin, M.; Santos, I.; Huang, V.; Juste, J.; Marzan, L.; Edirisinghe, M.; Stride, E. Effects of Gold Nanoparticles on the Stability of Microbubbles. Langmuir. 2012, 28, 13808-13815. 
39. Bain, C.; Manning-Benson, S.; Darton, R. Rates of Mass Transfer and Adsorption of CTAB at an Expanding Air-Water Interface. J. Colloid and Interface Sci. 2000, 229 (1), 247-256.

40. Breward, C.; Howell, P. The Drainage of a Foam Lamella. J. Fluid Mech. 2002, 458, 379-406.

41. Modini, R.; Russel, L.; Deane, G.; Stokes, M. Effect of Soluble Surfactant on Bubble Persistence and Bubble-Produced Aerosol Particles. J. Geophy. Res.; Atmospheres. 2013, 118, 1388-1400.

42. Kuo, Y.; Wang, C. Characteristics of Droplets Genereated By Bubble Bursting From Chromic Acid Solutions. J. Aerosol Sci. 1999, 30 (9), 1171-1179.

43. Jang, Y.; Park, S.; Char, K. Functionalization of Polymer Multilayer Thin Films for Novel Biomedical Applications. Korean J. Chem. Eng. 2011, 28, 5.

44. Roisman, T. Dynamics of Free Liquid Films During Formation of Polymer Foams. Colloids and Surfaces A: Physicochem. Eng. Aspects. 2011, 382, 113-117. 
For Table of Contents Only

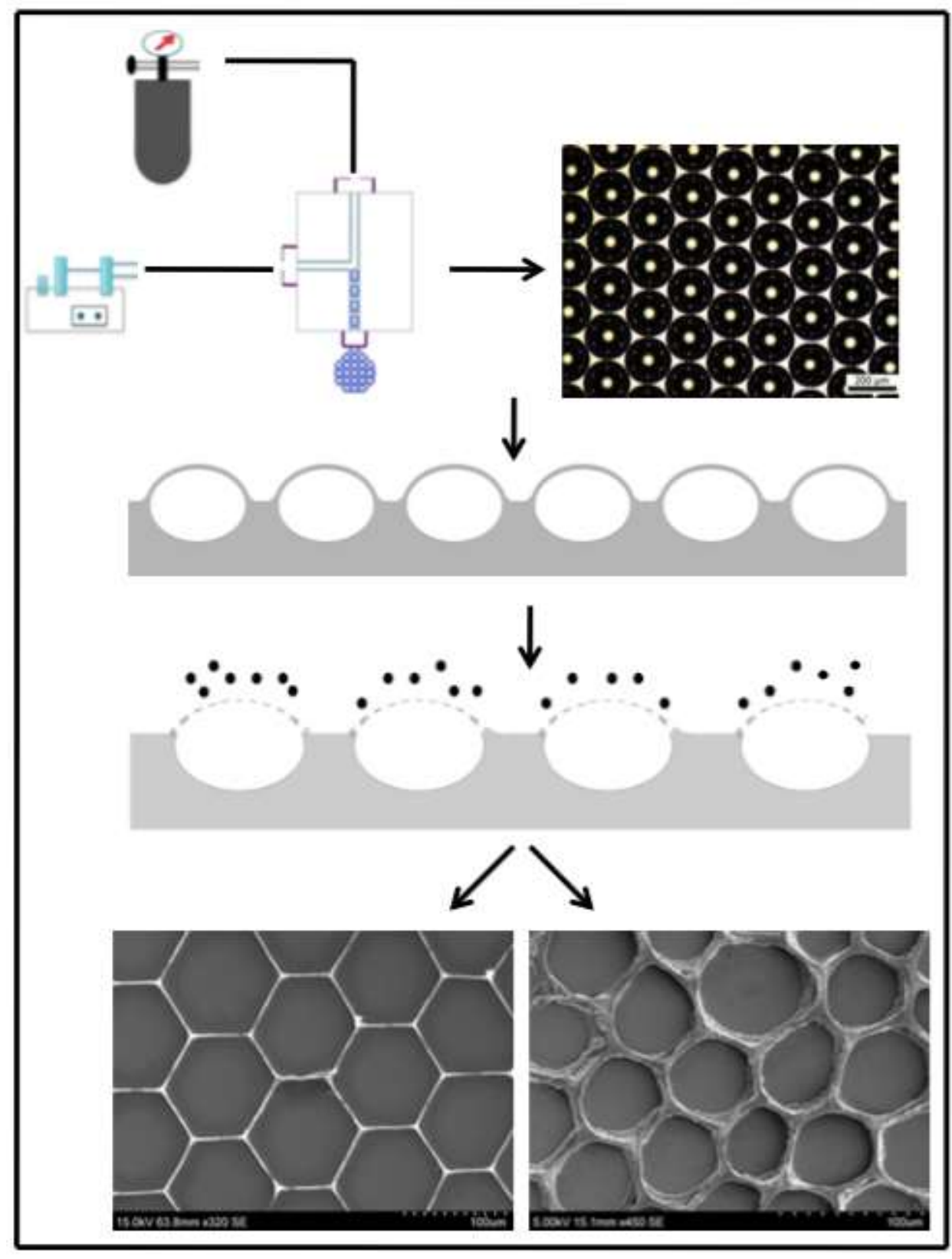

